\title{
A Genetic Algorithm to Obtain Consistency in Analytic Hierarchy Process
}

\author{
José Fabiano da Serra Costa \\ Rio de Janeiro State University (UERJ), Rio de Janeiro, Brazil
}

\begin{abstract}
This work presents a proposition to solve the problem of inconsistency in Analytic Hierarchy Process (AHP) matrices using genetic algorithms. Decision matrices resulting from an application of AHP can be considered an effective method to structure and represent relevant information of a strategic problem. Inconsistency in the results is a real and frequent possibility. In this case, the results obtained would become ineffective considering the objectives of the model, which means no gains in decision making. The Genetic Algorithms are probabilistic search computer models which are based on the mechanics of natural selection and genetics, combining the concepts of selective adaptation and survival of the fittest. They are considered to be a powerful technique of stochastic optimization and, probably the most important evolutionary computer techniques. Its application to the AHP matrices case allows the detection of inconsistent matrices, while offers alternative solutions to the decision-maker.
\end{abstract}

Keywords: AHP, Inconsistent matrices, Genetic algorithms.

\section{Introduction}

Whenever decision making is mentioned, the idea of something imprecise or undefined comes to mind. The human being has been confronting this questioning in every moment of his existence, whether it means an individual or a collective decision. Anyway, the essence of decision problems may be synthesized into the fact that expected results cannot be obtained unless one takes an action.

Considering decision making as an inevitable process, the need of creating methods of making this task easier to accomplish becomes necessary. In this context, due to economical and social environment complexity and to the vertiginous fall of the technological innovation, it becomes clear that the progress depends more and more on adopting planning and management innovative procedures. An answer to those needs is the decision making methods.

These methods are tools which involve modern techniques of informational systems, artificial intelligence, statistical quantitative methods, cognitive and behavioral psychology, among others, and they aim to offer the users favorable conditions to choose, minimizing the chances of mistake in decision making. Among the decision support methods, we point out the Multicriteria Decision Support Methodologies (Roy, 1985), with their schools. 
One of the most known and used multicriteria methods, based on Saaty's (1980) work, the Analytic Hierarchy Process - AHP is easily and successfully applied in specific cases of decision problems. AHP method is based on comparison matrices resulted from experts' opinion when comparing criteria (alternatives), in pairs.

However, it may occur that the final matrix is inconsistent, even if it represents the experts' opinion. The matrix consistency refers to necessary matrix properties such as transitivity and reciprocity. The inconsistency may occur for several reasons (Saaty, 1991). In such cases, the results obtained of each expert would be ineffective to the model objectives.

The aim of this paper is to use stochastic research methods known as Genetic Algorithms (Goldberg, 1989) to create alternative solutions. Considering the little consistency of a expert matrix, our goal becomes the attempt to create $n$ solutions (similar matrices), through an analysis of disturbs of the original matrix values.

\section{Analytic Hierarchy Process (AHP)}

As previously mentioned, the Analytic Hierarchy Process (AHP) developed by Saaty (1980) is probably the most used multicriteria methodology with several applications in different activities (Vaidya and Kumar, 2006). This method aims to select and choose alternatives, through a process that considers different evaluation criteria, and is based in three principles of analytical thinking: hierarchy construction, priority definition, logical consistency.

\section{Hierarchy Construction}

Problems, in AHP, are structured in hierarchic levels in order to search better comprehension and evaluation. Hierarchy construction is an elementary part of human rationalizing process. During this activity, people identify key elements to decision making, arranging them in affinity groups, which are organized in layers.

\section{Priority Definition}

The adjustment of AHP priorities is based on people's ability of acknowledging the relationship between observed situations and objects, comparing them in pairs in light of a specific focus or criteria (pairwise judgments).

\section{Logical Consistency}

In the method, it is possible to evaluate the resulting prioritization model according its consistency (Costa, 2006).

In the usage of AHP, it is necessary to define a global objective or main focus, identify the available alternatives (or choice possibilities) and select criteria and subcriteria (when necessary) to achieve the proposed objective. All theses elements must be hierarchically structured and the criteria selection must be complete, minimal and operational (Chankong and Haimes, 1983).

Considering the attributes $\mathrm{E}_{1}, \mathrm{E}_{2}, \ldots, \mathrm{E}_{\mathrm{n}}$, which contribute to reach a determined objective, the methodology proposes to compare the relative importance of each pair 
of attributes related to a superior hierarchic level. So, experts can contribute with an individual Judgment Matrix, like this $A=\left[a_{i j}\right]_{n x n}$, where $a_{i j}$ represents the relative importance of the attribute $\mathrm{E}_{\mathrm{i}}$ comparing to the attribute $\mathrm{E}_{\mathrm{j}}$, considering that $\mathrm{a}_{\mathrm{ij}}>1$, if and if only $E_{i}$ is more important than $E_{j}$, and that $a_{i j}=1 / a_{j i}$ for each pair $(i, j)$. Saaty (1991) proposed his own scale, named Fundamental Scale, to compare pairs of attributes.

Several alternatives to conjugate information given by evaluators have been proposed and many of those reached values very close to consistency (Forman and Peniwati, 2008; Costa and Belderrain, 2009; Ehrlich, 2004; Saaty and Peniwati, 2007; Freitas et al., 2008; Innes, 2008). However, the main aspect is to respect the basic properties of transitive and reciprocal matrices.

After the application of the method, once we have all the attributes and alternatives hierarchically distributed, what interests us is that the basic properties of the symmetrical matrix - reciprocity and transitivity - are sustained, as well as the consistency of the final result, and having $(n-1)$ comparisons we are able to deduct the others.

\section{Matrix Inconsistency}

Although pairwise judgments are based in the professionals' experience and knowledge, inconsistencies may happen, especially when dealing with a great number of judgments. Even with the most advanced instruments, it is difficult to obtain consistency in practice, which makes it necessary to have a method capable to evaluate the importance of this precision in a specific problem. In our case, what is called inconsistency is a violation of proportionality, which can sometimes mean the violation of transitivity. According to Saaty (1991), the consistency of a reciprocal and positive matrix is equivalent that its maximal eigenvalue (Graybill, 1983) equals the number of attributes involved in the model. This means that the closest the maximal eigenvalue $\left(\lambda_{\text {máx }}\right)$ reaches the number of attributes of the model $(n)$, the better will be the coherence of the judgments $\left(\lambda_{\text {máx }}=n\right)$.

It is also possible to estimate a consistency deviation from the results of the division of $\left(\lambda_{\text {máx }}-n\right)$ by $(n-1)$. Some authors (Dias et al., 1996) prefer to use the module of the difference between the number of attributes involved in the model and the maximal eigenvalue, $\left|n-\lambda_{\text {máx }}\right|$. Anyway, according to Saaty (1991) the measure of the poor consistency will be possible to estimate when compared to the value $\left(\lambda_{\text {máx }}-n\right) /(n-1)$, denominated Consistency Index $(C I)$, with values picked from random judgments and its correspondents in reverse positions of a matrix of the same size, with reciprocal matrices randomly generated (Saaty, 1991). This measure is referred to as Consistency Reason, so CR = CI / RI, where IR is the Random (Consistency) Index of a reciprocal and positive matrix randomly generated and must vary according to each matrix order. The closest this reason gets to zero, the more consistent the matrix will be. If the reason equals less than 0.10 , the consistency is considered satisfactory. A mathematical justification for considering this value satisfactory can be found in Vargas (1982).

However, it may occur that the final matrix, although representative of the experts opinion, is not consistent. This may happen due to failures in individual 
matrices or even by the accumulation of errors. Precisely, we refer here to matrices which consistency reason equals to a value greater than 0.10 .

An attempt of detecting and analyzing the inconsistency in matrices can be observed in Bramont (1996). The author presents ways to analyze the inconsistency of results obtained in applications of the AHP method, related to the usage of matrices in decision process.

\section{Genetic Algorithms}

The Genetic Algorithms (Holland, 1975) derive from the nature's evolution model. They are probabilistic research computational models that copy the natural evolution of the species, combining adaptation and survival concepts of the most capable individuals. They consist in groups of individuals and genetic operators that influence this population. They are computationally simple and powerful when it comes to optimal solutions (Eiben and Smith, 2003).

When compared to traditional optimization models, the Genetic Algorithms (GA) present advantages as: robusty, natural parallelism, independency, simplicity and integration (Dornellas, 1997; Mitsuo and Cheng, 2000). An additional advantage is the opportunity to model optimization problems for which there is no explicit objective function, requiring a simulation model to evaluate the performance of the candidate solution. Stopping rules definition is a possible obstacle when applying the GAs. The ideal situation would be stopping the algorithm whenever the optimal solution is obtained, however it could take excessive time and be little productive. So, the usual stopping rules are the maximum number of generations, the processing time limit and stopping when no substantial improvement happens after a few generations.

Although they are inherently random, the Genetic Algorithms utilize, in a very efficient way, the historical information existent in its code, in order to suggest new solutions, which improve the final result of the problem. Therefore, they are classified as emerging intelligence algorithms (Angeline, 1993). Holland (1975) proved that GAs cover the sample space in cubic order $\left(\mathrm{n}^{3}\right)$, which means, while the iterations grow in order $\mathrm{n}$ (which is computational interesting), the number of points covered is the sample space grows in $\mathrm{n}^{3}$.

GA consists of a cycle of the following stages: creation of a population of potential decoded solutions; evaluation of this population; selection of the most capable individuals; creation of a new population through genetic manipulation. As in the Natural Evolution Theory, GAs considers solutions that are a string of bits, which decode the parameters for the problem. The size of the population affects the efficiency of the algorithm. Each iteration of the algorithm is called a generation and both terms will be considered the same for this paper.

Genetic Algorithms provide solutions according to an evaluation function named Fitness Function - and to a series of operators (selection, reproduction, mutation and substitution). This function is a metric that diagnosis how adapted the individual is (Ashlock, 2005). The operator Selection chooses individuals to reproduce; in the Reproduction operator, there is the combination of two or more solutions to generate new ones; the Mutation operator is an alteration of bits intending to restore the population diversity; and the Substitution operator is responsible for replacing ancient 
solutions for the ones remaining from the reproduction and mutation processes (Gen and Cheng, 2000).

Some of the articles that use Analytic Hierarchy Process and Genetic Algorithms combined successfully are Yeh et al. (1999), Moneim (2008), Abessi et al. (2003), Xuesong et al. (2009), and Ge (2009).

\section{The Model}

Considering a matrix with CR greater than $10 \%$ resulted from an Analytic Hierarchy Process application, the aim of the system turns to be the generation of $n$ solutions with a CR lower or at least similar to $10 \%$. If this is achieved, the result will be consistent matrices similar to the original one. To this application, it should be considered matrices with variable inconsistency values, as long as they allow solutions of reasonable proximity to the original.

Taking a certain matrix, the program initially analyses the matrix consistency. It calculates the maximal eigenvalue, the consistency index and reason. If this reason is over $10 \%$, the matrix is considered inconsistent and the algorithm randomly generates a series of matrices with small disturbs in its values (avoiding the unitary diagonal) and sequentially generates an initial population. This population is submitted to the operators generating a new matrices population, which will have their evaluation function continually analyzed. This process is over when the program obtains matrices with CR lower than $10 \%$.

In this work, the adopted approach was to decode disturbs around the elements of a matrix so the number of bits necessary to decode a matrix is given by the number of its elements multiplied by the number of bits necessary to make disturbs in its elements. That considerably reduces the number of resultant bits from decoding a matrix. The number of bits to be used to decode and the range of disturbs in the matrix elements can be parameterized. Each individual of a certain generation is recomposed in the matrix structure. Then, the matrix $\mathrm{CR}$ and the degree of non reciprocity are calculated.

According to Mitsuo et al. (2006), the GA's structure and parameter settings affect its performance. In sequence, there is an analysis of the process steps, presenting in a proper way the operators as well as the beginning and end conditions, some comments and a simulation of the functioning. The procedures were programmed in $\mathrm{C}++$ and they were specifically designed for this work.

\section{Initialization}

The initial population of $\mathrm{n}$ individuals is randomly generated. This population can be parameterized. The generations occur with matrices composed of small disturbs, which should evolve naturally. Then, they should be added to the original matrix and have their consistency degree constantly evaluated. These are quadratic $\mathrm{n} \times \mathrm{n}$ matrices, where $\mathrm{n}$ varies from 2 to 9 . The limits imposed to the matrix dimensions ( 2 to 9 ), follow the orientations given by Saaty (1991). Notice that, similar to the biologic case, the evolution occurs only with diversity, so the greatest the variety of individuals in the initial population the easiest it will be to convert to the solution. 


\section{Evaluation}

The Fitness Function gives each individual the proper measure for the studied situation. This function would be represented like:

Fitness Function $=1-\mathrm{CR}$

The CR value varies from zero to one $(0.1)$ so the function would never be negative (as required by the algorithm procedure) and the greater is the CR value the more apt the individual would be. However, it was noticed that because the CR is considerably low, it is not enough to assure the matrix ideal conditions. So, the function has suffered a restrictive alteration. This restriction requires that all values of the matrix that disrespect the condition of anti-symmetry and the CR value to be below $10 \%$. The new function is now

Fitness Function $=1-(\mathrm{CR}+\mathrm{NR})$

and

$\mathrm{NR}=\mid 1-(\operatorname{Tr}+$ aij $\times$ aji $) \mid$

where $\mathrm{CR}=$ Consistency Reason, $\mathrm{NR}=$ Non Reciprocity, $\mathrm{Tr}=$ Tolerance Rate, aij = elements of line $\mathrm{i}$ and column $\mathrm{j}$. The Non Reciprocity (NR) works like a trigger from which the value is added to the function. To values of non reciprocity lower than the established limit, the Fitness Function is again evaluated as initially. This trigger condition allows to completely discard the influence of a tolerable non reciprocity in the Fitness Function, letting the algorithm evaluate the individuals in terms of its consistency reason up to a tolerable limit of non reciprocity. An output matrix is considered as solution as long as its Fitness Function, evaluated by the algorithm, is above the limit value established by the user.

\section{Selection}

This operator selects individuals which are apt to reproduce themselves. As a mechanism of Selection, the Raking with Roulette Wheel method, developed by Aguiar and Costa (1997), was used. According to the method, each individual is associated to a number or ranking $\mathrm{Ri}$, being $\mathrm{Ri}=(\mathrm{Ni} / \mathrm{N}) 2$, where $\mathrm{Ni}$ is the number of individuals with Fitness value lower than the lowest individual $\mathrm{i}$ and $\mathrm{N}$ is the total number of individuals in the population. After the definition of these numbers, the traditional Roulette Wheel method is used.

\section{Reproduction}

For the reproduction operator, it was used the Single Point (Mitchell, 1996) and a $10 \%$ rate of reproduction. However, both in the case of the reproduction as well as of the rate, the values can be chosen by the system operator, once the model is entirely parameterized.

\section{Mutation}

The mutation process is used to guarantee the diversity of the population. The question is to define the frequency it should occur. It was used a $10 \%$ probability or mutation rate. As in the reproduction case, this value can be altered at any moment. 


\section{Stopping Condition}

The algorithm stopping rule used requires the algorithm to stop searching when finding a solution or individual whose Fitness Function is lower than $10 \%$. So, the number of solutions is limited only by the user. However, as the model allows the user intervention, the algorithm can be stopped at any moment or a time limit can be established to find a solution.

\section{System Environment Description}

The system was developed in $\mathrm{C}++$, using a standard library, allowing a high degree of portability. It was used the integrated development environment Bloodshed Dev-C++, version 4, Mingw compiler 2.95.2 -1 and GNU Debugger 4.18 (GDB).

\section{Input Pre-requisites}

As requisites, the input matrix must be quadratic and diagonal. The system reads the matrix of text file, in the ascii format. The first line must contain the matrix dimension. The following lines must contain each of the matrix lines, with the elements separated by blanks.

\section{System Output}

The system shows in the standard output the results of the processing.

To flex the process of analysis and convergence of results, the system keeps some of the most influent items parameterized. Each parameter has a default value internally predefined. Optionally, it is possible to specify the values through the command line, such as the number of individuals of a population, the minimal quantity of individuals which are apt to characterize a solution, the minimal value above which an individual is considered apt and the number of bits to decode the disturbs.

\section{Example}

Random matrices with unitary diagonals and variable sizes were generated to be used as tests in this model. However, to compose the model evolution documentation, we used referenced matrices already studied in other situations.

The chosen matrices were extracted from Saaty (1991) because this study is one of the most detailed and complete when it comes to matrix inconsistency. These matrices vary in size and degree of inconsistency.

The solutions obtained were quite satisfactory and respected the model conditions, considering the involved parameters. Notice that in some cases, the size of the population is small and the system rapidly converges. In Table 1, the characteristics of the matrices are showed, including initial and final Consistency Reasons from the example matrices.

In matrix 2 (Saaty, 1991, pp. 33) the solution found by the system is quite interesting, considering that the initial matrix CR is relatively high (notice this is the highest number of generations). Specifically in the case of matrix 3 (Saaty, 1991, pp. 55), the high value of the initial CR was an obstacle to the final result. It suggests that high 
Table 1. Matrices characteristics.

\begin{tabular}{|c|c|c|c|c|c|c|}
\hline Matrix & Size & Population & Initial CR & Generations & Final CR & Fitness \\
\hline 1 & 3 & 100 & 0.116884 & 1380 & 0.0737133 & 0.901138 \\
\hline 2 & 6 & 100 & 0.238381 & 2262 & 0.0997367 & 0.900263 \\
\hline 3 & 4 & 500 & 0.532033 & 291 & 0.0905882 & 0.909412 \\
\hline 4 & 6 & 500 & 0.127419 & 117 & 0.0182739 & 0.981726 \\
\hline & & & & & 0.0920225 & 0.907978 \\
\cline { 5 - 7 } & 3 & 100 & 0.116884 & 492 & 0.0846125 & 0.915388 \\
\cline { 5 - 7 } & & & & & 0.0541502 & 0.903442 \\
\hline
\end{tabular}

initial CR values demand special treatment. Matrix 5 (Saaty, 1991, pp. 56) is, in fact, a variation of matrix 1, altering the stopping criteria of one of the three solutions. The solution matrices found by the system are plenty satisfactory and the CR values present small, non-meaningful variations. In matrices 3 and 4 (Saaty, 1991, pp. 119) cases, the size of the initial population was purposely raised, which would generally lead to more adapted solutions.

\section{Conclusions}

The main objective of this paper is to offer a possible solution to inconsistency in decision matrices. The approach involves Genetic Algorithms, which are introduced theoretically and practically (developed model). It is consensus, and a purpose of this study, that inconsistency in decision analysis must be treated in order to minimize the losses and make the decision process easier.

The importance of the Genetic Algorithms in this theme relates to the many advantages that this kind of computational method offers, among which the possibility of generating more than one solution to each matrix with an inconsistency problem, allowing the decision maker to have a greater number of options to replace the original matrix. On one hand, this subject may be seen as a complication, as in some circumstances the decision maker may not be interesting in choosing among various solutions close to the optimum. However, this becomes quite simple to resolve if the algorithm (model) is calibrated to offer only a solution for each problem.

There was a good performance of the algorithm model, requiring no user intervention and having no variations in the mutation and reproduction rates. Although these situations are very common in Genetic Algorithms, the system is still useful. It is worthy to notice the variation occurred in the reciprocity rate, according to the example, in order to keep solution similar to the original matrix. A necessary concern when using computational models is the time to process, a considerable factor in the problem solution evaluation. In this case, it was relatively short, considering the dimensions of the proposed problem, quite close to the expected time, which is an advantage of the model.

Attention to the fact that the models used as examples were all extracted of published cases. In most of these examples, it is satisfactory to respect the reciprocity condition, considering less important the transitivity condition. In fact, some authors 
consider that intransitivity among preferences may be considered as a natural phenomenon and not as a consequence of judgment errors.

\section{References}

Abessi, M.; Karimabad, A.H.; Faghih, N. and Sadeghieh, A. (2003) Integrating CSF, AHP and Genetic Algorithms for Information Systems Planning. Iranian Journal of Information Science \& Technology, Vol.1, No.1, pp. 16-29.

Aguiar, E. and Costa, J.F.S. (1997) Uma Implementação de Pesquisa Estocástica utilizando Algoritmos Evolutivos. Cadernos do IME, Vol. 1. (in Portuguese)

Angeline, P.J. (1993) Evolutionary Algorithms and Emergent Intelligence. Dissertation, The Ohio State University, USA.

Ashlock, D. (2005) Evolutionary Computation For Modeling and Optimization. London: Springer.

Bramont, B.P.P. (1996) Consideração de Incertezas nas Estimativas de Pesos dos Critérios em MCDM, in: Encontro Nacional de Engenharia de Produção - ENEGEP. (in Portuguese)

Chankong, Y. and Haimes, Y. (1983) Multiobjective Decision Making. Amsterdam: Ed. North Holland.

Costa, H.G. (2006) Auxílio Multicritério à Decisão: Método AHP. Rio de Janeiro: Latec/UFF - ABEPRO. (in Portuguese)

Costa, T.C. and Belderrain, M.C.N. (2009) Decisão em Grupo em Métodos Multicritério de Apoio à Decisão. Encontro de Iniciação Científica e Pós-Graduação do Instituto Tecnológico de Aeronáutica - ITA. (in Portuguese)

Dias, L.M.C.; Almeida, L.M.A.T. and Clímaco, J.C.N. (1996) Apoio Multicritério à Decisão. Coimbra: Universidade de Coimbra. (in Portuguese)

Dornellas, C.R.R. (1997) Otimização dos Despachos Reativos Utilizando Algoritmos Genéticos. Tese. Programa de Pós-Graduação em Engenharia, Universidade Federal do Rio de Janeiro, Rio de Janeiro, Brazil. (in Portuguese)

Ehrlich, P.J. (2004) Procedimentos para Apoio às Decisões: Gestão dos Riscos e de Objetivos Conflitantes. Escola de Administração de Empresas da Fundação Getúlio Vargas. (in Portuguese)

Eiben, A.E. and Smith, J.E. (2003) Introduction to Evolutionary Computing. London: Springer. Natural Computing Series.

Forman, E. and Peniwati, K. (1998) Aggregating Individual Judgments and Priorities with the Analytic Hierarchy Process. European Journal of Operacional Research, Vol. 108, No.1, pp. 165-169. http://dx.doi.org/10.1016/S0377-2217(97)00244-0

Freitas, A.L.P.; Trevizano, W.A. and Costa, H.G. (2008) Uma Abordagem Multicritério para Problemas Decisórios com Múltiplos Grupos de Avaliadores. Investigação Operacional, Vol. 28, pp. 133-149. (in Portuguese)

Ge, Y. (2009) Research on Green Suppliers' Evaluation Based on AHP \& Genetic Algorithm, in: International Conference on Signal Processing Systems, pp. 615-619.

Gen, M. and Cheng, R. (2000) Genetic Algorithms and Engineering Optimization. New York: Wiley.

Goldberg, D.E. (1989) Genetic Algorithms in search, optimization, and machine learning. Reading: Addison-Wesley.

Graybill, F.A. (1983) Matrices with Applications in Statistics. California: Wadsworth Inc. 
Holland, J. (1975) Adaptation in Natural and Artificial Systems. Michigan: The University of Michigan Press.

Innes, J. (2008) Managing Impact Reduction in Fisheries: A Multi-Criteria Assessment of Objective Priorities, in: Australian Conference of Economist (ACE08).

Mitchell, M. (1996) An Introduction to Genetic Algorithms. Cambridge: MIT Press.

Mitsuo, G. and Cheng, R. (2000) Genetic Algorithms and Engineering Optimization. New York: J. Wiley.

Mitsuo G.; Lin, L. and Jeongeun L. (2006) Interactive Adaptive Weight GA for Flexible Manufacturing System, in: Asia Pacific Industrial Engineering and Management Systems Conference.

Moneim, A.F.A. (2008) Fuzzy Genetic Prioritization in Multi-Criteria Decision Problems. Jordan Journal of Mechanical and Industrial Engineering, Vol. 2, No.4, pp. 175-182.

Roy, B. (1985) Methodologie Multicritere d'Aide á la Decision. Paris: Econômica.

Saaty, T.L. (1980) The Analytic Hierarchy Process. New York: Mc Graw Hill. Portuguese)

Saaty, T.L. (1991) Método de Análise Hierarquica. São Paulo: Makrom Books. (in

Saaty, T.L. and Peniwati, K. (2007) Group Decision Making: Drawing out and Reconciling Differences. Pittsburgh: RWS Publications.

Vaidya, O.S. and Kumar, S. (2006) Analytic Hierarchy Process: An overview of applications. European Journal of Operational Research, Vol. 169, No. 1, pp. 1-29. http://dx.doi. org/10.1016/j.ejor.2004.04.028

Vargas, L.G. (1982) Reciprocal Matrices with Random Coefficients. Mathematical model, Vol. 3, No. 1, pp. 69-81. http://dx.doi.org/10.1016/0270-0255(82)90013-6

Xuesong G.; Yiqiang W. and Liyan T. (2009) Machining Scheme Selection of Digital Manufacturing based on Genetic Algorithm and AHP. Journal of Intelligent Manufacturing, Vol. 20, No. 6, pp. 661-669. http://dx.doi.org/10.1007/s10845-008-0155-8

Yeh, J.M.; Lin, C.; Creng, B. and Gee, J.Y. (1999) A Modified Procedure Synthesizing Ratio Judgments in the AHP. The Journal of the Operational Research Society, Vol. 50, No .8, pp. 867-873.

\section{Biography}

José Fabiano da Serra Costa received his degree in Industrial Electric Engineering (1987) at CEFET - RJ, M.Sc. in Production Engineering (1990) at COPPE - UFRJ and D.Sc. in Production Engineering (2001) at COPPE - UFRJ. He is professor at Rio de Janeiro State University (UERJ). His research interests include Multicriteria Decision Methods, Genetic Algorithms, Probability and Statistics.

Contact: fabiano@ime.uerj.br

\section{Article Info:}

Received: March, 2011

Accepted: August, 2011 